\title{
Synthesis and photophysical properties of 1, 4-disubstituted naphthyloxymethyl- $N$-alkyl naphthimido-1,2,3-triazole
}

\author{
J RAMCHANDER, N RAMESHWAR, T SHESHASHENA REDDY, GAJULA RAJU and \\ A RAM REDDY* \\ Department of Chemistry, Osmania University, Hyderabad 500 007, India \\ e-mail: a_ramreddy@yahoo.com
}

MS received 3 December 2013; revised 13 February 2014; accepted 6 March 2014

\begin{abstract}
Regioselective synthesis of a series of 1,4-disubstituted of naphthoxymethyl-N-alkyl naphthalimide-1,2,3-triazoles employing click reaction is presented. Highly selective and efficient copper(I)-catalysed 1,3-dipolar cyclo addition between 1-naphthylpropargylic ether and azido alkyl naphthalimides yielded the title compounds in $74 \%$ to $94 \%$. The structure of all the new 1,2,3-triazoles was characterized by ${ }^{1} \mathrm{HNMR},{ }^{13} \mathrm{C}$ NMR, IR and Mass. The electronic absorption and emission studies revealed that the light absorbing and emitting chromophore is the naphthoxy moiety. There is no extensive delocalization of aromatic $\pi$-electrons in the active chromophore which exhibited lower quantum yields and lower Stokes shifts.
\end{abstract}

Keywords. Naphthylpropargylicether; azidonaphthalimides; 1,3-dipolar cycloaddition; regioselective.

\section{Introduction}

With increasing demand in developing fluorescent chemosensors for the detection of biological and environmental analytes, modular approaches have offered the possibility for rapidly constructing sensor molecular libraries. Among them, $\mathrm{Cu}(\mathrm{I})$-catalysed azidealkyne cyclo addition $(\mathrm{CuAAC})^{1,2}$ represents one of the most efficient approaches for chemical transformation to create new fluorescent molecules with desired spectral properties. ${ }^{3-13}$ The triazoles and substituted triazoles on conjugation with oxadiazoles were developed into organic light emitting diodes by Lee et al. ${ }^{14}$ who found that triazoles are efficient electron transporting molecules. Ruan and co-workers ${ }^{15}$ have investigated the chemo-sensing ability of 1,4 and 1,5 monoand bi-substituted 1,2,3-triazoles and found that when attached aromatic ring leads to extensive delocalization of $\pi$-electrons reducing the energy gap of $\pi-\pi^{*}$ transition. Further, they proved that these triazoles are potential ligands because of their N3, N3 and $\mathrm{C} 5$ are able to coordinate to metal centres. The ligand character of triazoles was further proved by Jia et al. who exploited the naphthol-triazole system for $\mathrm{Al}^{3+}$ detection, based on chelation-enhanced fluorescence technique. ${ }^{16}$ Czirok and co-workers ${ }^{17}$ investigated the aminophthimide-based three-armed fluorescent triazole receptors fluorsensing of the nucleoside

*For correspondence polyphosphate. Bag, Kundu and Talukdar ${ }^{18}$ demonstrated that click triazolenitrogens are binding sites for the selective recognition of metal ions. They have shown that triazolyl units are capable of installation of fluorescence properties into a non-fluorescent precursor and also electronic coupling between a triazole and a fluorophore gives rise to a modulated emission response to the fluorophore, solvatochromism and quantum yield. Triazole derivatives are known to exhibit various pharmacological properties such as anti-microbial, ${ }^{19}$ anti-inflammatory, ${ }^{20}$ cytotoxic, ${ }^{21}$ anticonvulsant, $^{22}$ analgesic ${ }^{23}$ and anti-viral. ${ }^{24}$ Triazoles have also been incorporated in a wide variety of therapeutically interesting drugs including $\mathrm{H} 1 / \mathrm{H} 2$ histamine receptor and antimycotic drugs such as fluconazole, itraconazole and voriconazole. On the other hand, heterocycles containing the naphthol nucleus also exhibit various biological activities. For instance, 2-naphthol derivatives have been reported as potential cognition enhancers. ${ }^{25}$ 2-Substituted-1-naphthol derivatives were shown to inhibit the activities of cyclooxygenase and 5-lipooxygenase. 1-Naphthol derivatives were found to possess potent anti-amnesic activity. Pharmacological importance and electronic properties of both triazoles and naphthol moiety have encouraged us to synthesize a series of novel 1,4-disubstituted naphthyloxy methyl-N-alkyl naphthimido-1,2,3-triazoles derivatives containing a 1,2,3-triazole. The synthetic route utilized the 'click reaction' which is a $\mathrm{Cu}(\mathrm{I})$-catalysed $[3+2]$ cycloaddition popularized by Sharpless as the 
key transformation to build up the triazole ring. ${ }^{26}$ Herein, we report the facile regioselective synthesis of a series of naphthol-based 1,2,3-triazoles employing click reaction and their electronic absorption and emission properties. Electronic absorption and emission studies revealed that the electron transporting ability of 1,4-disubstituted 1,2,3-triazoles did not give a new fluorophore as the conjugation between the two terminal substituents is prevented by the $\mathrm{sp}^{3}$-hybridized atoms such as carbon, nitrogen and oxygen.

\section{Experimental}

\subsection{Materials and apparatus}

Spectroscopic grade organic solvents were obtained from Finar Chemicals. Starting and other chemicals and reagents were purchased from Sigma-Aldrich unless otherwise stated and were used without further purification. Thin layer chromatography (TLC) was performed on silica gel $60 \mathrm{~F}_{254}$ aluminum plates (Merck). The reported melting points were uncorrected and determined in Polmon instrument (model No. MP-96). The IR spectra were recorded on Bruker Infrared model Tensor-27. ${ }^{1} \mathrm{H}$ NMR and ${ }^{13} \mathrm{C}$ NMR were recorded on a Bruker400 MHz Ultrashield spectrometer. The ESI mass spectra were recorded on a VG micro mass 7070-H. UV-vis spectra were recorded on Elico SL 159 UV-VIS spectrophotometer. Steady state fluorescence was investigated on Shimadzu RF-5301PC spectrofluorophotometer with $5 \mathrm{~nm}$ excitation and emission slit widths at $25^{\circ} \mathrm{C}$ employing $1 \mathrm{~cm}$ path length quartz cell. The relative fluorescence quantum yield, $\Phi$ is evaluated by employing Eq. 1 .

$$
\Phi_{\mathrm{unk}}=\Phi_{\mathrm{std}}\left(\frac{I_{\mathrm{unk}}}{I_{\mathrm{std}}}\right)\left(\frac{A_{\mathrm{std}}}{A_{\mathrm{unk}}}\right)\left(\frac{n_{\mathrm{unk}}}{n_{\mathrm{std}}}\right)^{2}
$$

where $\Phi, \Phi_{\text {std }}, \mathrm{I}_{\text {unk }}, \mathrm{I}_{\text {std }}, \mathrm{A}_{\text {std }}, \mathrm{A}_{\text {unk }}, \mathrm{n}_{\text {unk }}$ and $\mathrm{n}_{\text {std }}$ are fluorescence quantum yields, integral of the emission intensities, the absorbance at the excitation wavelength and refractive indexes of the corresponding solvents of the unknown samples and the standard, respectively. 9,10diphenyl anthracene was used as standard in cyclohexane solvent $(\Phi=0.9)$.

\subsection{General procedure for the synthesis of naphthylpropargylicethers (III1-2)}

Naphthylpropargylic ethers were synthesized starting from compound I as shown in scheme 1. Naphthylpropargylic ethers (III) were synthesized according to reported procedure. ${ }^{27}$

\subsection{General experimental procedure for the synthesis} of 1,4-disubstituted naphthyloxy methyl- $N$-alkyl naphthimido-1,2,3-triazoles.(IVI-2 a-f)

Compound $\mathrm{III}_{1-2}(2 \mathrm{mmol})$ and azides $\mathrm{IX}_{a-f}(2 \mathrm{mmol})$ were dissolved in $10 \mathrm{~mL}$ of DMF. To this mixture, $\mathrm{CuSO}_{4} .5 \mathrm{H}_{2} \mathrm{O}(25 \mathrm{mg}, 0.1 \mathrm{mmol})$ and sodium ascorbate
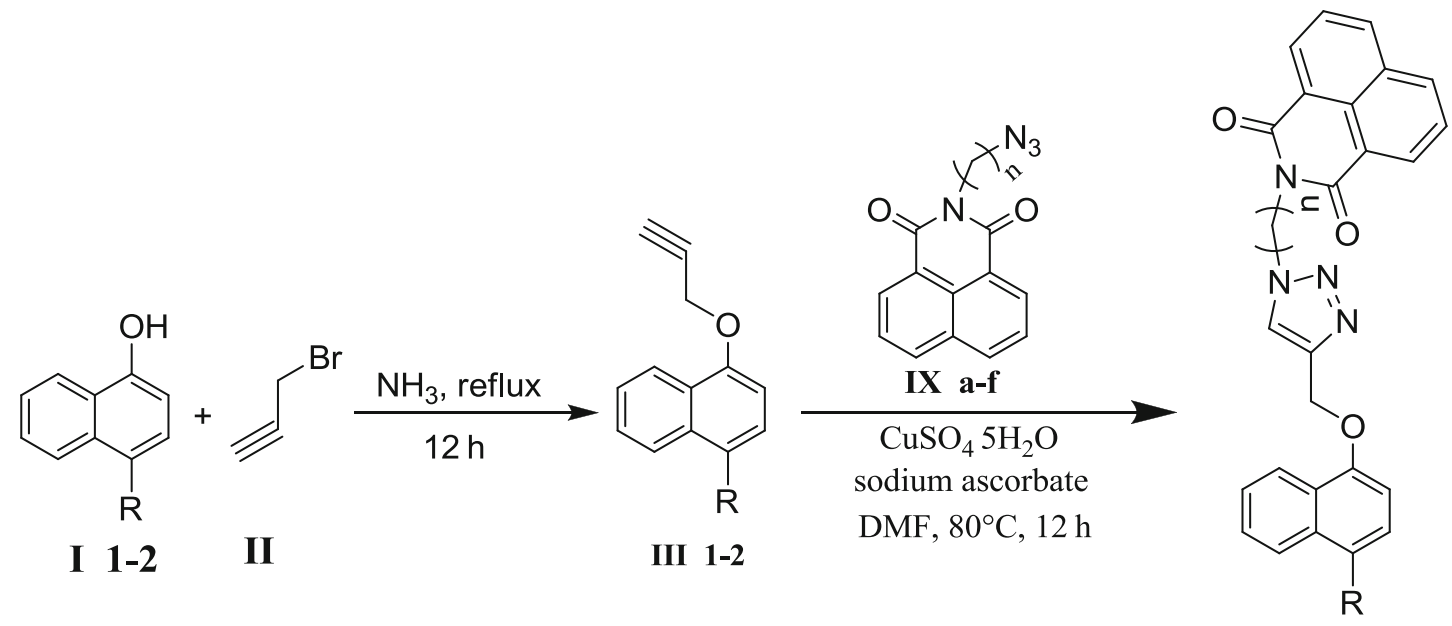

IV 1-a-f

IV 2-a-f

Here $\mathrm{R}=\mathrm{H}-1, \mathrm{R}=\mathrm{OCH}_{3}-2, \mathrm{n}=\mathrm{a}=2, \mathrm{~b}=3, \mathrm{c}=4, \mathrm{~d}=5, \mathrm{e}=6, \mathrm{f}=8$

Scheme 1. Synthesis of 1,4-disubstituted naphthyloxymethyl-N-alkylnaphthimido-1,2,3-triazoles. 
(39 mg, $0.2 \mathrm{mmol}$ ) aqueous solution were added and stirred at $80^{\circ} \mathrm{C}$ for $12 \mathrm{~h}$. The reaction mixture was poured into $25 \mathrm{~mL}$ of water and was extracted with $\mathrm{CHCl}_{3}(3 \times 25 \mathrm{~mL})$. The organic layers were combined, and washed with water $(20 \mathrm{~mL})$ and dried over anhydrous $\mathrm{Na}_{2} \mathrm{SO}_{4}$. The solvent was evaporated in vacuo to give the crude product. The crude product was purified by column chromatography using hexane: ethylacetate (9:1-6:4\%) as eluent to afford the pure product.

\subsection{General procedure for the synthesis of $N$-( $\omega$-azidoalkyl)-1,8-naphthalimide (IXa-f)}

One equivalent of $\mathrm{N}$-( $\omega$-bromoalkyl)-1,8-naphthalimide $\left(\mathrm{VIII}_{a-f}\right)$ was dissolved in DMF mix and two equivalents of sodium azide were added to it. The mixture was stirred at $80^{\circ} \mathrm{C}$ for $12 \mathrm{~h}$. After completion of the reaction, DMF was removed in vacuo and poured into ice cold water, extracted with chloroform. After evaporation of chloroform, the pure compound was collected.

\subsection{General procedure for the synthesis of $N$-( $\omega$-bromoalkyl)-1,8-naphthalimide (VIIIa-f)}

To a solution compound VI ( $0.5 \mathrm{gm}, 1 \mathrm{mmol})$ in acetonitrile $(30 \mathrm{~mL})$, anhydrous potassium carbonate $(553 \mathrm{mg}$, $4 \mathrm{mmol}$ ) and terminal dibromoalkane (561 mg, $3 \mathrm{mmol}$ ) were added and the mixture was refluxed for $12 \mathrm{~h}$. After completion of the reaction, anhydrous potassium carbonate was removed by filtration and the solvent was evaporated under reduced pressure to get the crude product. This was further purified by column chromatography (10\% EtOAc-hexane) to afford the compound VIII a-f.

\subsection{Synthesis of 1H-benzo}

[de]isoquinoline-1,3(2H)-dione (VI)

1,8-Naphthalic anhydride (V)(1 g, $5.07 \mathrm{mmol})$ was taken in ammonia solution $(60 \mathrm{~mL})$ and stirred at $100^{\circ} \mathrm{C}$ for $12 \mathrm{~h}$. After cooling, a yellowish solid was obtained, to this $200 \mathrm{~mL}$ of ice cold water was added and filtered at pump. The solid product was dried in oven at $100^{\circ} \mathrm{C}$. The compound is characterized by IR, ${ }^{1} \mathrm{HNMR}$ and mass spectra.

\subsection{Spectral data}

2.7a 1H-benzo [de]isoquinoline-1,3(2H)-dione (VI): Yield 91.7\%; M.p. 262-264 ${ }^{\circ} \mathrm{C}$; IR (KBr): 3440, 3059, 1701, 1676, 1622, $1586 \mathrm{~cm}^{-1}$. ${ }^{1} \mathrm{HNMR}\left(\mathrm{CDCl}_{3}\right.$,
$400 \mathrm{MHz}) \delta 7.79$ (t, 2H), 8.45-8.43 (m, 4H), 11.71 (bs, $1 \mathrm{H}, \mathrm{D}_{2} \mathrm{O}$ exchanged). ESI-MS: $m / z$ 198[M+H $]^{+}$.

\section{$2.7 \mathrm{~b}$ 2-(4-bromobutyl)-1H-benzo[de]isoquinoline-}

1,3(2H)-dione (VIII c): Yield 88.4\%; M.p. $100-103^{\circ} \mathrm{C}$; IR (KBr): 3095, 1697, 1664, $1588 \mathrm{~cm}^{-1} .{ }^{1} \mathrm{HNMR}$ $\left(\mathrm{CDCl}_{3}, 400 \mathrm{MHz}\right): 1.87-2.03(\mathrm{~m} 4 \mathrm{H}), 3.47(\mathrm{t}, 2 \mathrm{H})$, $4.23(\mathrm{t}, 2 \mathrm{H}), 7.75(\mathrm{t}, J=8 \mathrm{~Hz}, 2 \mathrm{H}), 8.21(\mathrm{~d}, J=8.4 \mathrm{~Hz}$, $2 \mathrm{H}), 8.60(\mathrm{~d}, J=7.2 \mathrm{~Hz}, 5 \mathrm{H}) \cdot{ }^{13} \mathrm{CNMR}$ (DMSO$\left.d_{6}, 400 \mathrm{MHz}\right) \delta 164.15,133.96,131.54,131.25,128.10$, $126.93,122.53,39.34,33.18,30.25,26.89$. ESI-MS: $\mathrm{m} / \mathrm{z} 332[\mathrm{M}+\mathrm{H}]^{+}$.

\section{7c 2-(5-bromopentyl)-1H-benzo[de]isoquinoline-}

1,3(2H)-dione (VIII d): Yield 87.2\%; M.p. 118$120^{\circ} \mathrm{C}$; IR (KBr): 3060, 2954, 1695, 1660, 1589, $1512 \mathrm{~cm}^{-1} .{ }^{1} \mathrm{HNMR}\left(\mathrm{CDCl}_{3}, 400 \mathrm{MHz}\right) \delta 1.58-1.63$ $(\mathrm{m}, 2 \mathrm{H}), 1.75-1.83(\mathrm{~m}, 2 \mathrm{H}), 1.93-2.00(\mathrm{~m}, 2 \mathrm{H}), 3.45$ $(\mathrm{t}, J=6.8 \mathrm{~Hz}, 2 \mathrm{H}), 4.21(\mathrm{t}, J=7.6 \mathrm{~Hz}, 2 \mathrm{H}), 7.78$ $(\mathrm{t}, J=7.6 \mathrm{~Hz}, 2 \mathrm{H}), 8.23(\mathrm{~d}, J=8.0 \mathrm{~Hz}, 2 \mathrm{H}), 8.62$ (d, $J=7.4 \mathrm{~Hz}, 2 \mathrm{H})$. ESI-MS: $m / z: 346[\mathrm{M}+\mathrm{H}]^{+}$.

2.7d 2-(6-bromohexyl)-1H-benzo[de]isoquinoline-1,3 (2H)-dione (VIII e): Yield 85.8\%; M.p. 90-94 ${ }^{\circ} \mathrm{C}$; IR (KBr): 3061, 2954, 2932, 1694, 1662, 1587, $1512 \mathrm{~cm}^{-1} .{ }^{1} \mathrm{HNMR}\left(\mathrm{CDCl}_{3}, 400 \mathrm{MHz}\right) \delta 1.41-1.59$ $(\mathrm{m}, 4 \mathrm{H}), 1.69-1.79(\mathrm{~m}, 2 \mathrm{H}), 1.84-1.93(\mathrm{~m}, 2 \mathrm{H}), 3.37$ $(\mathrm{t}, J=6.79 \mathrm{~Hz}, 2 \mathrm{H}), 4.14(\mathrm{t}, J=7.6 \mathrm{~Hz}, 2 \mathrm{H}), 7.73$ $(\mathrm{t}, J=7.93 \mathrm{~Hz}, 2 \mathrm{H}), 8.16(\mathrm{~d}, J=8.03 \mathrm{~Hz}, 2 \mathrm{H}), 8.55$ $(\mathrm{d}, J=7.36 \mathrm{~Hz}, 2 \mathrm{H}) .{ }^{13} \mathrm{CNMR}$ (DMSO- $d_{6}, 400 \mathrm{MHz}$ ) $\delta=26.39,28.01,32.87,33.28,40.33,123.19,126.92$, 128.44, 131.10, 133.65, 164.14. ESI-MS: m/z360 $[\mathrm{M}+\mathrm{H}]^{+}$.

2.7e 2-(8-bromooctyl)-1H-benzo[de]isoquinoline-1,3 (2H)-dione (VIII f): Yield 80.6\%: M.p. 70$75^{\circ} \mathrm{C}$; IR (KBr): 3061, 2934, 1697, 1661, 1588, $1510 \mathrm{~cm}^{-1} .{ }^{1} \mathrm{HNMR}\left(\mathrm{CDCl}_{3}, 400 \mathrm{MHz}\right) \delta 1.30-1.44$ $(\mathrm{m}, 8 \mathrm{H}), 1.69-1.77(\mathrm{~m}, 2 \mathrm{H}), 1.80-1.87(\mathrm{~m}, 2 \mathrm{H}), 3.39(\mathrm{t}$, $J=6.8 \mathrm{~Hz}, 2 \mathrm{H}), 4.17(\mathrm{t}, J=7.6 \mathrm{~Hz}, 2 \mathrm{H}), 7.75(\mathrm{t}, J=$ $7.2 \mathrm{~Hz}, 2 \mathrm{H}), 8.20(\mathrm{~d}, J=8.0 \mathrm{~Hz}, 2 \mathrm{H}), 8.59(\mathrm{~d}, J=$ $7.2 \mathrm{~Hz}, 2 \mathrm{H}$ ). ${ }^{13} \mathrm{CNMR}$ (DMSO- $\left.d_{6}, 400 \mathrm{MHz}\right) \delta 26.93$, $28.01,28.06,28.55,29.02,32.77,33.58,40.34,122.91$, $126.82,128.20,131.02,131.63,133.61,164.06$. ESI-MS: $m / z 374[\mathrm{M}+\mathrm{H}]^{+}$.

2.7f $N$-( $\omega$-azidoalkyl)-1, 8-naphthalimide (IXb): M.p. $128^{\circ} \mathrm{C} ;{ }^{1} \mathrm{HNMR}\left(\mathrm{CDCl}_{3}, 400 \mathrm{MHz}\right) \delta 2.09(\mathrm{~m}, 2 \mathrm{H})$, $3.45(\mathrm{t}, J=6.8 \mathrm{~Hz}, 2 \mathrm{H}), 4.36(\mathrm{t}, J=6.4 \mathrm{~Hz}, 2 \mathrm{H}), 7.78$ $(\mathrm{t}, J=7.6 \mathrm{~Hz}, 2 \mathrm{H}), 8.23\left(\mathrm{dd}, J^{1}=1.2 \mathrm{~Hz}, J=8.4 \mathrm{~Hz}\right.$, 
2H), $8.61\left(\mathrm{dd}, J^{1}=0.8 \mathrm{~Hz}, J=7.2 \mathrm{~Hz}, 2 \mathrm{H}\right)$. ESI-MS: $\mathrm{m} / \mathrm{z} 303[\mathrm{M}+\mathrm{Na}]^{+}$.

$2.7 \mathrm{~g} \quad 2-(2-(4(($ naphthalen-1-yloxy)methyl $)-1 H-1,2,3-$ triazol-1-yl)ethyl)-1H-benzo[de]isoquinoline-1,3(2H)dione $\left(I V_{1-a}\right)$ : M.p. $166-168^{\circ} \mathrm{C}$; IR $(\mathrm{KBr})$ : 3067, 2958, 2871, 1991,1953,1908, 1698, 1652,1623, $1588,1512,1438,1406,1380,1347,1321,1260$, 1234, 1177, 1144, 1079, $1032 \mathrm{~cm}^{-1} \cdot{ }^{1} \mathrm{H}$ NMR (DMSO- $\left.d_{6}, 400 \mathrm{MHz}\right): \delta 4.1(\mathrm{t}, J=6.8 \mathrm{~Hz}, 2 \mathrm{H}), 4.8(\mathrm{t}$, $J=6 \mathrm{~Hz}, 2 \mathrm{H}), 4.9(\mathrm{~s}, 2 \mathrm{H}), 6.8(\mathrm{~d}, J=7.6 \mathrm{~Hz}, 1 \mathrm{H}), 6.9$ $(\mathrm{d}, J=7.2 \mathrm{~Hz}, 1 \mathrm{H}), 7.5(\mathrm{t}, J=2 \mathrm{~Hz}, 2 \mathrm{H}), 7.8(\mathrm{t}, J=$ $7.6 \mathrm{~Hz}, 2 \mathrm{H}), 8.1(\mathrm{t}, J=6 \mathrm{~Hz}, 1 \mathrm{H}), 8.2(\mathrm{~s}, 1 \mathrm{H}), 8.3(\mathrm{~d}$, $J=6 \mathrm{~Hz}, 2 \mathrm{H}), 8.4(\mathrm{~d}, J=7.6 \mathrm{~Hz}, 2 \mathrm{H}), 8.4(\mathrm{~d}, \mathrm{~J}=$ $7.2 \mathrm{~Hz}, 2 \mathrm{H}$ ) ${ }^{13} \mathrm{CNMR}$ (DMSO- $\left.d_{6}, 400 \mathrm{MHz}\right) \delta 40.17$, $42.38,60.02,110.90,111.71,122.41,122.81,125.68$, $126.78,127.96,129.41,131.03,131.38,131.92$, $133.92,135.78,136.75,139.30,139.52,147.92$, 158.21, 164.41. ESI-MS:m/z $449[\mathrm{M}+\mathrm{H}]^{+}$.

2.7h 2-(3-(4-((naphthalen-1-yloxy)methyl)-1H-1,2,3triazol-1-yl)propyl)-1H-benzo[de]isoquinoline-1,3(2H)dione $\left(I V_{1-b}\right)$ : M.p. $76-78^{\circ} \mathrm{C}$; IR (KBr): 3441, 3163, 3053, 2946, 2870, 2600, 2508, 2413, 2265, 2094, 1957, 1871, 1776, 1701, 1656, 1580, 1509, 1460, 1386, 1340, 1270, 1237, 1155, 1097, $1052 \mathrm{~cm}^{-1} .{ }^{1} \mathrm{H}$ NMR (DMSO$\left.d_{6,}, 400 \mathrm{MHz}\right) \delta 2.5(\mathrm{~m}, 2 \mathrm{H}), 4.81(\mathrm{t}, J=6.8 \mathrm{~Hz}, 2 \mathrm{H}), 4.5$ $(\mathrm{t}, J=7.2 \mathrm{~Hz}, 2 \mathrm{H}) 5.3(\mathrm{~s}, 2 \mathrm{H}), 7.1(\mathrm{~d}, J=7.2 \mathrm{~Hz}, 1 \mathrm{H})$, $7.4(\mathrm{~d}, J=7.2 \mathrm{~Hz}, 1 \mathrm{H}), 7.5(\mathrm{t}, J=2 \mathrm{~Hz}, 2 \mathrm{H}), 7.8(\mathrm{t}, J=$ $7.6 \mathrm{~Hz}, 2 \mathrm{H}), 8.1(\mathrm{t}, J=6 \mathrm{~Hz}, 1 \mathrm{H}), 8.3(\mathrm{~d}, J=6 \mathrm{~Hz}$, $2 \mathrm{H}), 8.3(\mathrm{~s}, 1 \mathrm{H}), 8.4(\mathrm{~d}, J=8.4 \mathrm{~Hz}, 2 \mathrm{H}), 8.5(\mathrm{~d}, J=$ $6.8 \mathrm{~Hz}, 2 \mathrm{H}) .{ }^{13} \mathrm{CNMR}$ (DMSO- $\left.d_{6}, 400 \mathrm{MHz}\right) \delta 30.42$, 52.94, 54.51, 67.03, 110.99, 111.07, 125.51, 126.79, $127.36,129.53,129.65,130.13,130.50,131.35$, $131.66,132.65,135.87,136.47,139.25,139.46$, 147.93, 158.74, 168.78, ESI-MS: $m / z, 462[\mathrm{M}+\mathrm{H}]^{+}$.

$2.7 \mathrm{i} \quad 2-(4-(4-(($ naphthalen-1-yloxy)methyl $)-1 H-1,2,3-$ triazol-1-yl)butyl)-1H-benzo[de]isoquinoline-1,3(2H)dione $\left(I V_{1-c}\right)$ : M.p. $78-80^{\circ} \mathrm{C}$; IR (KBr): 3142, 3059, 2921, 2852, 1716, 1615, 1509, 1441, 1390, 1268, 1238, $1147,1097,1070 \mathrm{~cm}^{-1} .{ }^{1} \mathrm{HNMR}$ (DMSO- $d_{6}, 400 \mathrm{MHz}$ ) $\delta 1.8(\mathrm{~m}, 2 \mathrm{H}), 2.1(\mathrm{~m}, 2 \mathrm{H}), 4.0(\mathrm{t}, J=6 \mathrm{~Hz}, 2 \mathrm{H}) 4.4$ $(\mathrm{t}, J=6.8 \mathrm{~Hz}, 2 \mathrm{H}), 5.4(\mathrm{~s}, 2 \mathrm{H}), 6.1(\mathrm{~s}, 1 \mathrm{H}), 6.8(\mathrm{~m}$, $2 \mathrm{H}), 6.9(\mathrm{~d}, J=7.6 \mathrm{~Hz}, 1 \mathrm{H}), 7.4(\mathrm{~m}, 5 \mathrm{H}), 7.7(\mathrm{~s}, 1 \mathrm{H})$, $7.8(\mathrm{~d}, J=7.6 \mathrm{~Hz}, 1 \mathrm{H}), 8.0(\mathrm{~s}, 1 \mathrm{H}), 8.2(\mathrm{~d}, J=8 \mathrm{~Hz}$, $2 \mathrm{H}$ ). ${ }^{13} \mathrm{CNMR}$ (DMSO- $\left.d_{6}, 400 \mathrm{MHz}\right) \delta 25.99,27.00$, 31.93, 50.07, 67.42, 101.27, 105.38, 111.85, 112.38, $113.58,120.82,121.96,125.27,125.56,125.62$,
$125.86,126.48,127.49,134.49,152.67,153.91$, 155.11, 161.25, 161.69. ESI-MS: $m / z 477[\mathrm{M}+\mathrm{H}]^{+}$.

$2.7 \mathrm{j} 2-\{5-[4-($ naphthalen-1-yloxymethyl)-[1,2,3]triazollyl]pentyl]-benzo[de] isoquinoline-1,3-dione $\left(I V_{1-d}\right)$ : M.p. $108-110^{\circ} \mathrm{C}$; IR (KBr): 3141, 3057, 2951, 2872, 2598, 2119, 1957, 1873, 1778, 1697, 1658, 1591, 1509, 1437, 1342, 1240, 1154, $1067 \mathrm{~cm}^{-1} .{ }^{1} \mathrm{HNMR}$ (DMSO$\left.d_{6,} 400 \mathrm{MHz}\right): \delta=1.3(\mathrm{~m}, 2 \mathrm{H}) 1.7(\mathrm{~m}, 2 \mathrm{H}), 1.9(\mathrm{~m}, 2 \mathrm{H})$, $4.0(\mathrm{t}, J=7.6 \mathrm{~Hz}, 2 \mathrm{H}), 4.4(\mathrm{t}, J=7.2 \mathrm{~Hz}, 2 \mathrm{H}), 5.3(\mathrm{~s}$, $2 \mathrm{H}), 7.0(\mathrm{~d}, J=7.2 \mathrm{~Hz}, 1 \mathrm{H}), 7.1(\mathrm{~d}, J=7.2 \mathrm{~Hz}, 1 \mathrm{H})$, 7.4(t, $J=8.4 \mathrm{~Hz}, 1 \mathrm{H}), 7.5(\mathrm{t}, J=2.4 \mathrm{~Hz}, 2 \mathrm{H}), 7.8(\mathrm{t}$, $J=8.4 \mathrm{~Hz}, 2 \mathrm{H}), 8.12(\mathrm{~d}, J=8 \mathrm{~Hz}, 2 \mathrm{H}), 8.3(\mathrm{~s}, 1 \mathrm{H})$, $8.4(\mathrm{~d}, J=8 \mathrm{~Hz}, 2 \mathrm{H}), 8.5(\mathrm{~d}, J=7.2 \mathrm{~Hz}, 2 \mathrm{H}) .{ }^{13} \mathrm{CNMR}$ $\left(\mathrm{DMSO}-d_{6}, 400 \mathrm{MHz}\right) \delta 28.62,32.13,34.65,54.49$, $61.08,67.00,83.66,110.96,125.50,125.86,127.12$, $130.50,130.70,131.15,131.33,132.31,132.45$, $132.64,132.72,135.85,136.40,139.23,139.42$, 158.70, 168.52. ESI-MS: $\mathrm{m} / z 491[\mathrm{M}+\mathrm{H}]^{+}$.

$2.7 \mathrm{k} \quad 2-(6-(4-(($ naphthalen-1-yloxy)methyl)-1H-1,2,3triazol-1yl)hexyl)-1H- benzo[de]isoquinoline-1,3(2H)dione $\left(I V_{1-e}\right):$ M.p. $98-100^{\circ} \mathrm{C}$; IR (KBr): 3058, 3012, 2938, 2861, 1697, 1658, 1625, 1587, 1508, 1460, $1440,1390,1346,1268,1236,1175,1098,1069$, $1021 \mathrm{~cm}^{-1} .{ }^{1} \mathrm{HNMR}$ (DMSO- $\left.d_{6}, 400 \mathrm{MHz}\right) \delta=1.2(\mathrm{~m}$, 2H) $1.3(\mathrm{~m}, 2 \mathrm{H}), 1.6(\mathrm{~m}, 2 \mathrm{H}), 1.8(\mathrm{~m}, 2 \mathrm{H}) 4.0(\mathrm{t}, J=$ $7.2 \mathrm{~Hz}, 2 \mathrm{H}), 4.4(\mathrm{t}, J=6.8 \mathrm{~Hz}, 2 \mathrm{H}), 5.3(\mathrm{~s}, 2 \mathrm{H}), 7.1(\mathrm{~d}$, $J=6.8 \mathrm{~Hz}, 1 \mathrm{H}), 7.4(\mathrm{t}, J=8.0 \mathrm{~Hz}, 1 \mathrm{H}), 7.1(\mathrm{t}, J=$ $2.4 \mathrm{~Hz}, 2 \mathrm{H}), 7.8(\mathrm{t}, J=8.0 \mathrm{~Hz}, 2 \mathrm{H}), 8.1(\mathrm{~d}, J=$ $8.4 \mathrm{~Hz}, 2 \mathrm{H}), 8.1(\mathrm{~d}, J=8.4 \mathrm{~Hz}, 1 \mathrm{H}), 8.3(\mathrm{~s}, 1 \mathrm{H}), 8.4$ $(\mathrm{d}, J=8.0 \mathrm{~Hz}, 2 \mathrm{H}), 8.4(\mathrm{~d}, \mathrm{~J}=7.2 \mathrm{~Hz}, 2 \mathrm{H}) .{ }^{13} \mathrm{CNMR}$ $\left(\right.$ DMSO- $\left.d_{6}, 400 \mathrm{MHz}\right) \delta 27.33,27.55,30.00,31.12$, $54.81,60.94,66.98,110.37,125.36,125.42,125.74$, $126.61,126.79,127.20,130.06,130.20,130.71$, $131.38,132.30,132.64,135.71,136.33,138.99$, 139.14, 139.17, 158.70. ESI-MS:m/z $505[\mathrm{M}+\mathrm{H}]^{+}$.

2.71 2-\{8-[4-(naphthalen-1-yloxymethyl)-[1,2,3]triazol$1 y l$ loctyl]benzo[de] isoquinoline-1,3-dione $\left(I V_{1-f}\right)$ :: M.p. $78-80^{\circ} \mathrm{C}$; IR (KBr): 3050, 2929, 2851, 2599, 2417, 2118, 1968, 1914, 1872, 1786, 1698, 1662, 1591, 1508, 1438, 1345, 1267, 1238, 1154, 1098, $1051 \mathrm{~cm}^{-1} .{ }^{1} \mathrm{HNMR}$ (DMSO-d $\left.d_{6}, 400 \mathrm{MHz}\right) \delta 1.2(\mathrm{~m}$, $8 \mathrm{H}) 1.6(\mathrm{~m}, 2 \mathrm{H}), 1.8(\mathrm{~m}, 2 \mathrm{H}), 4.0(\mathrm{t}, J=7.6 \mathrm{~Hz}, 2 \mathrm{H})$ $4.3(\mathrm{t}, J=7.2 \mathrm{~Hz}, 2 \mathrm{H}), 5.3(\mathrm{~s}, 2 \mathrm{H}), 7.0(\mathrm{~d}, J=7.6 \mathrm{~Hz}$, $1 \mathrm{H}), 7.1(\mathrm{~d}, J=7.6 \mathrm{~Hz}, 1 \mathrm{H}), 7.4(\mathrm{t}, J=2.4 \mathrm{~Hz}, 1 \mathrm{H}), 7.5$ $(\mathrm{t}, J=2.4 \mathrm{~Hz}, 2 \mathrm{H}), 7.8(\mathrm{t}, J=7.6 \mathrm{~Hz}, 2 \mathrm{H}), 8.1(\mathrm{~d}, J=$ $8.4 \mathrm{~Hz}, 2 \mathrm{H}), 8.3(\mathrm{~s}, 1 \mathrm{H}), 8.4(\mathrm{~d}, J=7.6 \mathrm{~Hz}, 2 \mathrm{H}), 8.4$ $(\mathrm{d}, J=7.2 \mathrm{~Hz}, 2 \mathrm{H}){ }^{13} \mathrm{CNMR}$ (DMSO- $d_{6}, 400 \mathrm{MHz}$ ) $\delta 31.02,31.63,32.65,33.46,33.73,34.92,54.79$, 
$60.95,66.96,110.41,125.36,125.74,126.79,127.24$, $130.09,130.19,130.29,130.91,131.32,131.87$, $132.30,132.64,135.71,136.36,139.02,139.16$, 158.70, 168.49. ESI-MS: $m / z 533[\mathrm{M}+\mathrm{H}]^{+}$

$2.7 \mathrm{~m}$ 2-(2-(4((4-methoxynaphthalen-1-yloxy)methyl)1H-1,2,3-triazol-1-yl)ethyl)-1H-benzo[de]isoquinoline1,3(2H)-dione $\left(I V_{2-a}\right)$ : M.p. $120-122^{\circ} \mathrm{C}$; IR ( $\left.\mathrm{KBr}\right)$ : 2923, 2853, 1696, 1658, 1624, 1591, 1462, 1440, 1381, 1344, 1272, 1236, 1152, 1095, $1048 \mathrm{~cm}^{-1} .{ }^{1} \mathrm{HNMR}$ $\left(\mathrm{DMSO}-d_{6}, 400 \mathrm{MHz}\right) \delta 3.6(\mathrm{~d}, J=4.4,2 \mathrm{H}), 3.9(\mathrm{~s}, 3 \mathrm{H})$ $4.1(\mathrm{~d}, J=6.8 \mathrm{~Hz}, 2 \mathrm{H}), 4.9(\mathrm{~s}, 2 \mathrm{H}), 6.8(\mathrm{~d}, J=7.6 \mathrm{~Hz}$, $1 \mathrm{H}), 6.9(\mathrm{~d}, J=7.2 \mathrm{~Hz}, 1 \mathrm{H}), 7.5(\mathrm{t}, J=2 \mathrm{~Hz}, 2 \mathrm{H})$, $7.8(\mathrm{t}, J=7.6 \mathrm{~Hz}, 2 \mathrm{H}), 8.1(\mathrm{~d}, J=6 \mathrm{~Hz}, 2 \mathrm{H}), 8.2$ $(\mathrm{s}, 1 \mathrm{H}), 8.4(\mathrm{~d}, J=7.6 \mathrm{~Hz}, 2 \mathrm{H}), 8.4(\mathrm{~d}, J=6 \mathrm{~Hz}$, $2 \mathrm{H}){ }^{13} \mathrm{CNMR}$ (DMSO- $\left.d_{6}, 400 \mathrm{MHz}\right) \delta$ 47.03, 60.45, $61.28,63.58,107.92,110.61,122.41,125.68,126.47$, $126.59,127.42,129.41,130.68,130.85,131.00$, 131.82, 132.80, 135.71,136.36, 138.93, 151.75, 154.56, 168.83. ESI-MS: $m / z, 479[\mathrm{M}+\mathrm{H}]^{+}$.

2.7n 2-(3-(4((4-methoxynaphthalen-1-yloxy)methyl)-1H1,2,3-triazol-1-yl)propyl)-1H-benzo[de] isoquinoline-1,3 $(2 H)$-dione $\left(I V_{2-b}\right)$ : M.p. 58-60 ${ }^{\circ} \mathrm{C}$; IR $(\mathrm{KBr}): 3076$, 2949, 2861, 2725, 2598, 2002, 1979, 1880, 1695, $1655,1588,1442,1389,1348,1272,1236,1172,1096$, $1057 \mathrm{~cm}^{-1} .{ }^{1} \mathrm{HNMR}$ (DMSO- $\left.d_{6}, 400 \mathrm{MHz}\right) \delta 2.5(\mathrm{~m}$, $2 \mathrm{H}), 3.5(\mathrm{t}, J=6.4 \mathrm{~Hz}, 2 \mathrm{H}), 3.9(\mathrm{~s}, 3 \mathrm{H}), 4.1(\mathrm{t}, J=$ 7.6, 2H), $5.3(\mathrm{~s}, 2 \mathrm{H}), 7.1(\mathrm{~d}, J=7.2 \mathrm{~Hz}, 1 \mathrm{H}), 7.4(\mathrm{~d}$, $J=7.2 \mathrm{~Hz}, 1 \mathrm{H}), 7.5(\mathrm{t}, J=2 \mathrm{~Hz}, 2 \mathrm{H}), 7.8(\mathrm{t}, J=$ $7.6 \mathrm{~Hz}, 2 \mathrm{H}), 8.3(\mathrm{~d}, J=6 \mathrm{~Hz}, 2 \mathrm{H}), 8.3(\mathrm{~s}, 1 \mathrm{H}), 8.4$ $(\mathrm{d}, J=8.4 \mathrm{~Hz}, 2 \mathrm{H}), 8.5(\mathrm{~d}, J=7.2 \mathrm{~Hz}, 2 \mathrm{H}) .{ }^{13} \mathrm{CNMR}$ $\left(\right.$ DMSO- $\left.d_{6}, 400 \mathrm{MHz}\right) \delta 27.26,39.58,42.76,54.55$, $64.22,104.25,104.38,110.90,111.15,125.45,126.60$, $129.60,130.25,131.27,132.33,132.45,135.81$, $136.41, \quad 139.39,139.45, \quad 147.90,158.46,168.55$. ESI-MS: $m / z 493[\mathrm{M}+\mathrm{H}]^{+}$.

2.7o 2-(4-(4-((4-methoxynaphthalen-yloxy)methyl)-1H1,2,3-triazol-1-yl)butyl)-1H-benzo[de]isoqunolene-1,3 $(2 \mathrm{H})$-dione $\left(I V_{2-c}\right):$ M.p. $63-65^{\circ} \mathrm{C}$; IR $(\mathrm{KBr}): 3074$, 2939, 2873, 2498, 2403, 2095, 1954, 1716, 1614, 1510, $1462,1388,1272,1146,1071 \mathrm{~cm}^{-1} .{ }^{1} \mathrm{H}$ NMR (DMSO$\left.d_{6}, 400 \mathrm{MHz}\right) \delta 1.7(\mathrm{~m}, 2 \mathrm{H}), 2.0(\mathrm{~m}, 2 \mathrm{H}), 3.9(\mathrm{~s} 3 \mathrm{H}), 4.1$ $(\mathrm{t}, J=1.6 \mathrm{~Hz}, 2 \mathrm{H}) 4.4(\mathrm{t}, J=1.6 \mathrm{~Hz}, 2 \mathrm{H}), 5.2(\mathrm{~s}, 2 \mathrm{H})$, $6.1(\mathrm{~s}, 1 \mathrm{H}), 6.8(\mathrm{~d}, J=8.4 \mathrm{~Hz}, 1 \mathrm{H}), 6.9(\mathrm{~m}, 3 \mathrm{H}), 7.0(\mathrm{~d}$, $J=8.8 \mathrm{~Hz}, 1 \mathrm{H}), 7.5(\mathrm{t}, J=3.6 \mathrm{~Hz}, 2 \mathrm{H}), 7.6(\mathrm{~d}, J=$ $8.8 \mathrm{~Hz}, 2 \mathrm{H}), 8.1(\mathrm{~d}, J=2 \mathrm{~Hz}, 2 \mathrm{H}), 8.3(\mathrm{~s}, 1 \mathrm{H}), 8.3(\mathrm{~s}$, $1 \mathrm{H}) .{ }^{13} \mathrm{CNMR}$ (DMSO- $\left.d_{6}, 400 \mathrm{MHz}\right) \delta$ 23.32, 30.91, 31.68, 54.29, 60.74, 67.30, 106.31, 108.83, 110.92, $116.31,117.55,118.26,126.60,126.75,130.95$,
$131.03,131.06,131.52,152.56,154.17,158.47$, 159.93, 165.34, 166.77, 166.81. ESI-MS: $\mathrm{m} / \mathrm{z} 507$ $[\mathrm{M}+\mathrm{H}]^{+}$.

2.7p 2-(5-(4-((4-methoxynaphthalen-yloxy)methyl)-1H1,2,3-triazol-1-yl)pentyl)-1H-benzo[de]isoqunolene-1,3 $(2 H)$-dione $\left(I V_{2-d}\right)$ : M.p. $108-110^{\circ} \mathrm{C}$; IR (KBr): 3131 , 3067, 2947, 2853, 1950, 1781, 1696, 1658, 1592, 1463, $1388,1272,1237,1099,1049 \mathrm{~cm}^{-1} .{ }^{1} \mathrm{H}$ NMR (DMSO$\left.d_{6}, 400 \mathrm{MHz}\right) \delta 1.3(\mathrm{~m}, 2 \mathrm{H}) 1.7(\mathrm{~m}, 2 \mathrm{H}), 1.9(\mathrm{~m}, 2 \mathrm{H}), 3.9$ $(\mathrm{s}, 3 \mathrm{H}), 4.0(\mathrm{t}, J=7.2 \mathrm{~Hz}, 2 \mathrm{H}) 4.4(\mathrm{t}, J=6.8 \mathrm{~Hz}, 2 \mathrm{H})$, $5.2(\mathrm{~s}, 2 \mathrm{H}), 6.8(\mathrm{~d}, J=8.4 \mathrm{~Hz}, 1 \mathrm{H}), 7.0(\mathrm{~d}, J=8.4 \mathrm{~Hz}$, $1 \mathrm{H}), 7.5(\mathrm{t}, J=4 \mathrm{~Hz}, 2 \mathrm{H}), 7.8(\mathrm{t}, J=7.2 \mathrm{~Hz}, 2 \mathrm{H}), 8.0$ $(\mathrm{d}, J=1.6 \mathrm{~Hz}, 2 \mathrm{H}), 8.3(\mathrm{~s}, 1 \mathrm{H}), 8.4(\mathrm{~d}, J=8.4 \mathrm{~Hz}$, $4 \mathrm{H}) .{ }^{13} \mathrm{CNMR}$ (DMSO- $\left.d_{6}, 400 \mathrm{MHz}\right) \delta 28.61,32.12$, 34.65 , 54.46, 60.76, 61.35, 67.29, 108.89, 110.91, $126.57,126.64,126.77,127.14,130.68,130.91$, $131.07,131.10,131.28,132.33,132.47,135.86$, $136.41,139.43,152.54,154.13,168.53$. ESI-MS: $\mathrm{m} / \mathrm{z}$ $521[\mathrm{M}+\mathrm{H}]^{+}$.

2.7q 2-(6-(4-((4-methoxynaphthalen-1-yloxy)methyl)1H-1,2,3-triazol-1-yl)hexyl)-1H-benzo[de]isoqunolene1,3(2H)-dione $\left(I V_{2-e}\right)$ : M.p. $98-100^{\circ} \mathrm{C}$; IR (KBr): 3069, 2934, 2859, 2599, 2423, 2309, 1959, 1699, 1657, 1591, 1463, 1439, 1387, 1272, 1237, 1098, 1078, $1023 \mathrm{~cm}^{-1} .{ }^{1} \mathrm{HNMR}$ (DMSO- $\left.d_{6}, 400 \mathrm{MHz}\right) \delta 1.3(\mathrm{~m}$, 2H) $1.3(\mathrm{~m}, 2 \mathrm{H}), 1.6(\mathrm{~m}, 2 \mathrm{H}), 1.8(\mathrm{~m}, 2 \mathrm{H}) 3.9(\mathrm{~s}, 3 \mathrm{H})$, $4.0(\mathrm{t}, J=7.2 \mathrm{~Hz}, 2 \mathrm{H}), 4.4(\mathrm{t}, J=6.8 \mathrm{~Hz}, 2 \mathrm{H}), 5.2$ $(\mathrm{s}, 2 \mathrm{H}), 6.8(\mathrm{~d}, J=8.4 \mathrm{~Hz}, 1 \mathrm{H}), 7.0(\mathrm{~d}, J=8.4 \mathrm{~Hz}, 1 \mathrm{H})$, $7.4(\mathrm{t}, J=3.2 \mathrm{~Hz}, 2 \mathrm{H}), 7.8(\mathrm{t}, J=7.2 \mathrm{~Hz}, 2 \mathrm{H}), 8.0$ $(\mathrm{d}, J=2.4 \mathrm{~Hz}, 2 \mathrm{H}), 8.3(\mathrm{~s}, 1 \mathrm{H}), 8.4(\mathrm{~d}, J=8.4 \mathrm{~Hz}, 2 \mathrm{H})$, $8.5(\mathrm{~d}, J=6.4 \mathrm{~Hz}, 2 \mathrm{H}) .{ }^{13} \mathrm{CNMR}$ (DMSO- $\left.d_{6,} 400 \mathrm{MHz}\right)$ $\delta 30.76,31.12,32.52,34.77,54.69,60.56,61.37$, $67.40,108.35,108.47,110.64,110.96,126.52,126.69$, $127.21,129.52,130.72,130.78,131.02,132.01$, $132.60,135.75,136.41,139.18,152.59,154.21$, 168.47. ESI-MS:m/z $535[\mathrm{M}+\mathrm{H}]^{+}$.

2.7r 2-(8-(4-((4-methoxynaphthalen-1-yloxy)methyl)1H-1,2,3-triazol-1-yl)octyl)-1H-benzo[de]isoqunolene1,3(2H)-dione $\left(I V_{2-f}\right)$ : M.p. $108-110^{\circ} \mathrm{C}$; IR ( $\left.\mathrm{KBr}\right)$ : 2919, 2851, 1697, 1659, 1592, 1462, 1386, 1345, $1271,1237,1156,1096,1024 \mathrm{~cm}^{-1} .{ }^{1} \mathrm{HNMR}$ (DMSO$d_{6,400 \mathrm{MHz})} \delta 1.2(\mathrm{~m}, 8 \mathrm{H}) 1.6(\mathrm{~m}, 2 \mathrm{H}), 1.8(\mathrm{~m}, 2 \mathrm{H})$, $3.9(\mathrm{~s}, 3 \mathrm{H}), 4.0(\mathrm{t}, J=7.6 \mathrm{~Hz}, 2 \mathrm{H}) 4.3(\mathrm{t}, J=7.2 \mathrm{~Hz}$, $2 \mathrm{H}), 5.2(\mathrm{~s}, 2 \mathrm{H}), 6.8(\mathrm{~d}, J=8.4 \mathrm{~Hz}, 1 \mathrm{H}), 7.0(\mathrm{~d}, J=$ $8.4 \mathrm{~Hz}, 1 \mathrm{H}), 7.4(\mathrm{t}, J=0.8 \mathrm{~Hz}, 2 \mathrm{H}), 7.8(\mathrm{t}, J=7.6 \mathrm{~Hz}$, $2 \mathrm{H}), 8.0(\mathrm{~d}, J=4.8 \mathrm{~Hz}, 2 \mathrm{H}), 8.3(\mathrm{~s}, 1 \mathrm{H}), 8.4(\mathrm{~d}, J=$ $8.0 \mathrm{~Hz}, 2 \mathrm{H}), 8.4(\mathrm{~d}, J=6.8 \mathrm{~Hz}, 2 \mathrm{H}) .{ }^{13} \mathrm{CNMR}$ (DMSO$d_{6,400 \mathrm{MHz})} \delta 30.97,31.62,32.60,33.41,33.73$, 
$34.86,54.57,60.75,67.29,108.87,110.95,111.27$, $126.56,126.74,127.20,129.50,130.67,130.92$, $131.04,131.08,131.29,132.37,132.49,135.88$, 136.45, 139.45, 152.51, 154.12, 168.54. ESI-MS:m/z $563[\mathrm{M}+\mathrm{H}]^{+}$.

\section{Results and discussion}

The present investigation focuses on the development of a few triazolonaphthalenes starting from 1-naphthol. The synthetic chemistry employed to prepare the target compounds is outlined in scheme 1 . Regioselective synthesis of 1, 2, 3-triazole-substituted bisnaptholotriazoles involves three steps: (1) Propargylation of naphthol, (2) Synthesis of alkyl azides (scheme 2) and (3) Click reaction of propargylated naphthols with azides (scheme 1 and table 1).

Naphthols I1-2 on reaction with propargyl bromide in presence of $\mathrm{K}_{2} \mathrm{CO}_{3}$ give propargylatednapthyl ethers III1-2. The structure of naphthylether was confirmed by appearance of propargylic and terminal alkyne protons at $\delta 2.5$ and 4.8 in the ${ }^{1} \mathrm{H}$ NMR spectrum. The IR spectra of these compounds exhibited a new weak absorption maximum at around $2100 \mathrm{~cm}^{-1}$ characteristic of $\lambda_{\mathrm{c} \equiv \mathrm{c}}$ stretching indicating the formation of propargylated naphthol ethers III $1-2$.

$\mathrm{N}$-( $\omega$-bromo)alkyl)-1,8-naphthalimides $\left(\mathrm{VIII}_{\mathrm{a}-\mathrm{f}}\right)$ were freshly generated by the reaction of naphthalimide with appropriate alkyldibromides in acetonitrile and anhydrous potassium carbonate with good yields. Completion of the reaction was monitored by thin layer chromatography.

$\mathrm{N}$-( $\omega$-Azido)alkyl)-1,8-naphthalimides (IXa-f) were freshly prepared by the reaction of appropriate $\mathrm{N}-(\omega$ bromo)alkyl)-1,8-naphthalimide (VIIIa-f) with sodium azide in DMF with good yields.

The final step of the synthesis was the click reaction. Propargylic ethers of naphthalols (III1-2) react with azidonaphthalimides (IXa-f) in presence of aq. $\mathrm{CuSO}_{4} \cdot 5 \mathrm{H}_{2} \mathrm{O}$ and aq. sodium ascorbate in DMF to give 1,4-disubstituted naphthyloxy methyl-N- alkyl naphthimido-1,2,3-triazoles (IV1-2a-f) in excellent yields. The structures of all compounds were characterized by ${ }^{1} \mathrm{H},{ }^{13} \mathrm{C}$ NMR spectra and mass spectra. Formation of triazole ring was unequivocally established by the characteristic chemical shift value of the triazolyl proton resonated at $\delta 8.07-8.54 \mathrm{ppm}$. Triazole ring formation can also be confirmed by ${ }^{13} \mathrm{C}$ spectra with the new signals of the olefinic C-atoms of the 1,2,3-triazole moiety at $\delta 122.5-127 \mathrm{ppm}$ and $\delta$ 139-148.

\subsection{Electronic spectral study of 1,4-disubstituted naphthyloxy methyl $N$-alkyl naphthimido-1,2,3-triazoles}

Formation of 1,2,3-triazole moiety extended the aromatic conjugation between electron donor and electron acceptor molecules. It was demonstrated that 1,4disubstituted triazoles give enhanced spectral properties when triazole ring extends the aromatic delocalization from the donor moiety to the acceptor through intramolecular charge transfer phenomena $1,2,5$. If it is assumed that when the triazole is placed in between two aromatic chromophores and prevent the conjugation by introducing $\mathrm{sp}^{3}$-hybridized atoms then the resulting molecule may yield no better spectral properties. The 1,4-disubstituted naphthoxymethyl$\mathrm{N}$-alkyl naphthalimide-1,2,3-triazoles reported in this study possess no possibility for the electron delocalization from naphthyl to naphthalimide or vice versa via 1,2,3-triazole ring due to the presence of $\mathrm{sp}^{3}$-hybridized linker atoms such as oxygen, nitrogen and carbon. It is therefore interesting to investigate the electronic absorption and emission properties in order to evaluate aromatic conjugation from one end to another (figure 1).

\subsection{Electronic absorption spectral study}

Electronic absorption spectra of IV 2c, IV $2 \mathrm{~d}$ and IV 2e in DMF are shown in figure 1. Similarly, electronic

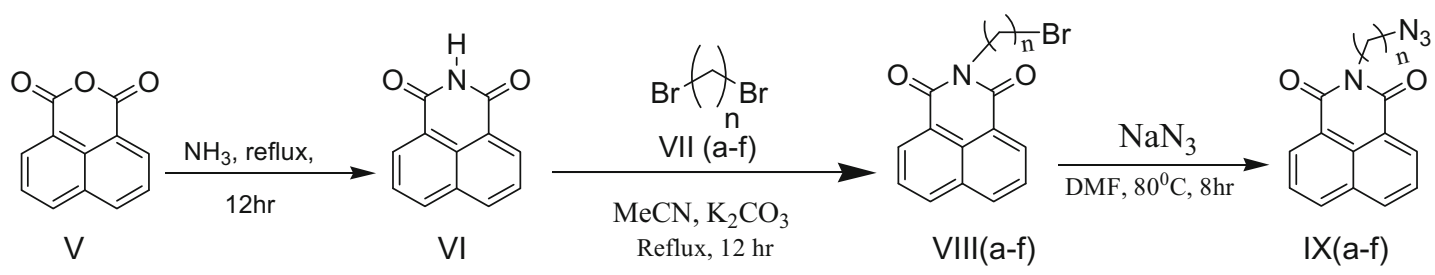

Where $\mathrm{n}=2-\mathrm{a}, 3-\mathrm{b}, 4-\mathrm{c}, 5-\mathrm{d}, 6-\mathrm{e}, 8-\mathrm{f}$.

Scheme 2. Synthesis of azidonapthalimides. 
Table 1. Synthesis of 1,4-disubstituted naphthyloxymethyl-N-alkyl naphthimido-1,2,3-triazoles.

\begin{tabular}{lccccc}
\hline S. no. & Compound & $\mathrm{R}$ & $N$ & Time (h) & Yield (\%) \\
\hline 1 & IV 1a & $\mathrm{H}$ & 2 & 12.0 & 82 \\
2 & IV 1b & $\mathrm{H}$ & 3 & 12.0 & 86 \\
3 & IV 1c & $\mathrm{H}$ & 4 & 12.0 & 92 \\
4 & IV 1d & $\mathrm{H}$ & 5 & 12.0 & 79 \\
5 & IV 1e & $\mathrm{H}$ & 6 & 12.0 & 88 \\
6 & IV 1f & $\mathrm{H}$ & 8 & 12.0 & 90 \\
7 & IV 2a & $\mathrm{OCH}_{3}$ & 2 & 12.0 & 79 \\
8 & IV 2b & $\mathrm{OCH}_{3}$ & 3 & 12.0 & 82 \\
9 & IV 2c & $\mathrm{OCH}_{3}$ & 4 & 12.0 & 90 \\
10 & IV 2d & $\mathrm{OCH}_{3}$ & 5 & 12.0 & 79 \\
11 & IV 2e & $\mathrm{OCH}_{3}$ & 6 & 12.0 & 12.0 \\
12 & IV 2f & $\mathrm{OCH}_{3}$ & 8 & & 94 \\
\hline
\end{tabular}

absorption spectra of 2-(3-(4((4-methoxynaphthalen-1yloxy)methyl)-1H-1,2,3-triazol-1-yl)propyl)-1H-benzo [de]isoquinoline-1,3(2H)-dione, IV $2 \mathrm{~b}$ in four different solvents are given in figure 2. Electronic absorption spectral data of all the 1,4-disubstituted naphthoxymethyl-N-alkyl naphthalimide-1,2,3-triazoles including the starting naphthols and their propargyl ethers is shown in tables 2 to 6.

From figure 1 it can be noticed that electronic transition that occurred are due to the naphthalene aromatic ring. Further, these transitions occur due to the naphthoxy moiety. The ${ }^{1} \mathrm{La}$ transition of naphthalene occurs at longer wavelength while the ${ }^{1} \mathrm{Lb}$ transition occurred at shorter wavelength. However, from molar extinction coefficients, it can be revealed that the triazole ring contributed significantly to the spectral shifts. Attachment of triazole caused a little bathochromic shift of naphthol or naphthoxypropargyl

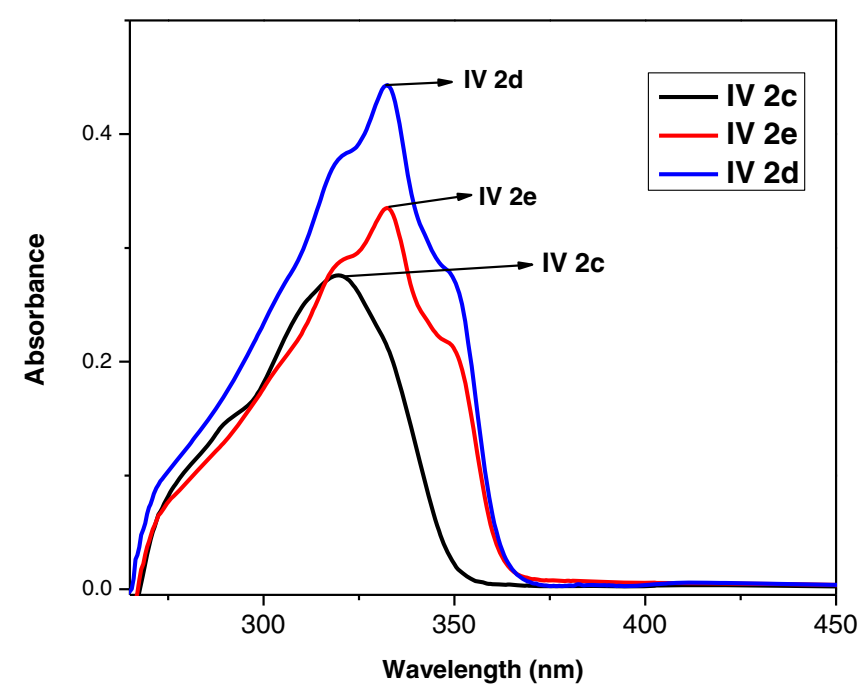

Figure 1. Electronic absorption spectrum of IV 2 c $1.32 \times$ $10^{-5} \mathrm{M}$, IV $2 \mathrm{~d} 1.41 \times 10^{-5} \mathrm{M}$ and IV $2 \mathrm{e} 1.06 \times 10^{-5} \mathrm{M}$ in DMF. ether in 1,4-disubstituted naphthoxymethyl-N-alkyl naphthalimide-1,2,3-triazoles. Similarly, covalent attachment of triazolyl unit to the alkoxy carbon of naphthyl ether enhanced the absorptivity of 1,4disubstituted naphthoxymethyl-N-alkyl naphthalimide1,2,3-triazoles.

Examination of figure 2 reveals that absorption spectrum of the compound IV $2 b$ in water is different from that of rest of the solvents. In water, compound IV $2 b$ exhibited a single broad absorption maximum, while in the remaining solvents, it has given a doublet absorption maxima in the near visible region. Similar observation was found in all the 1,4-disubstituted naphthoxymethyl$\mathrm{N}$-alkyl naphthalimide-1,2,3-triazoles. On closer examination of the absorption spectral data presented in tables 2 to 6 , it can be observed that there is no extensive delocalization of aromatic $\pi$-elecrons in the 1,4disubstituted naphthoxymethyl-N-alkyl naphthalimide1,2,3-triazoles.

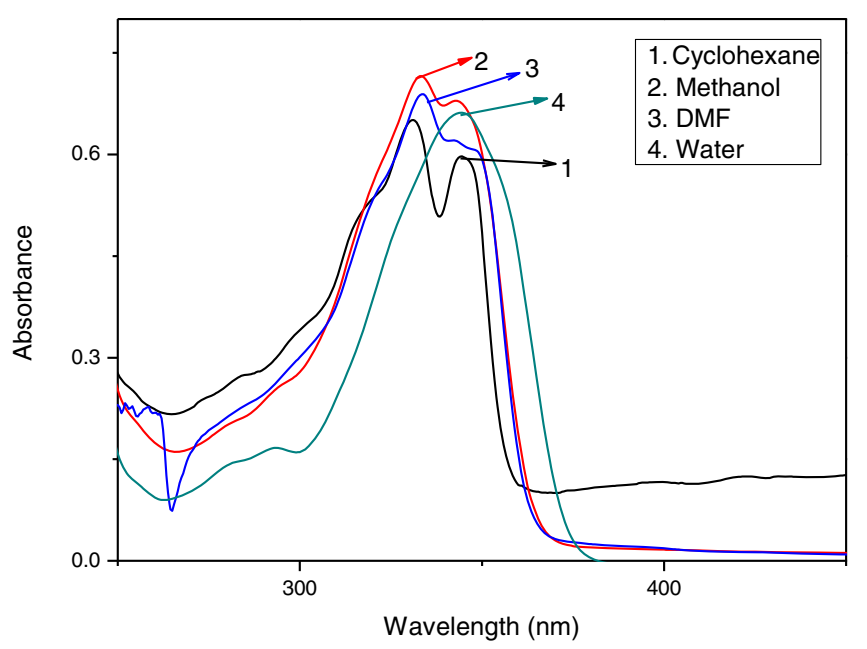

Figure 2. Electronic absorption spectrum of IV 2 b $2.08 \times$ $10^{-5} \mathrm{M}$ in different solvents. 
Table 2. Electronic absorption and fluorescence data of $\alpha$-naphthol, 4-methoxy naphthol, naphthylpropargylicethers, 1,4disubstituted naphthyloxymethyl-N-alkyl naphthimido-1,2,3-triazoles in cyclohexane.

\begin{tabular}{|c|c|c|c|c|c|}
\hline Compound & $\lambda_{\mathrm{abs} \cdot \max }(\mathrm{nm})\left(\in, \mathrm{dm}^{2} \mathrm{~mol}^{-1}\right)$ & $\lambda_{\text {ex }}(\mathrm{nm})$ & $\lambda_{\text {flu.max }}(\mathrm{nm})$ & Stokes shift (nm) & $\Phi_{\mathrm{f}}$ \\
\hline I -1 & $309\left(4.3 \times 10^{3}\right), 323\left(3.53 \times 10^{3}\right)$ & 369 & 452 & 83 & $8 \times 10^{-6}$ \\
\hline I -2 & $333\left(1.16 \times 10^{4}\right), 369\left(1.93 \times 10^{3}\right)$ & 369 & 452 & 83 & $2.55 \times 10^{-3}$ \\
\hline III -1 & $320\left(4.3 \times 10^{1}\right), 379\left(4.2 \times 10^{2}\right)$ & 369 & 453 & 84 & $1.3 \times 10^{-7}$ \\
\hline III -2 & $307\left(7.6 \times 10^{3}\right), 315\left(7.6 \times 10^{3}\right), 330\left(7.5 \times 10^{3}\right)$ & 369 & 453 & 84 & $3.6 \times 10^{-5}$ \\
\hline IV $1 \mathbf{b}$ & $\begin{array}{l}283\left(4 \times 10^{4}\right), 312\left(3.7 \times 10^{4}\right), 320\left(3.72 \times 10^{4}\right) \\
331\left(3.71 \times 10^{4}\right), 342\left(3.74 \times 10^{4}\right), 368\left(3.25 \times 10^{4}\right)\end{array}$ & 369 & 453 & 84 & $5.57 \times 10^{-2}$ \\
\hline IV 1c & $\begin{array}{l}290\left(2.43 \times 10^{4}\right), 309\left(2.52 \times 10^{4}\right), 320\left(2.51 \times 10^{4}\right) \\
345\left(2.1 \times 10^{4}\right)\end{array}$ & 368 & 453 & 85 & $2.2 \times 10^{-3}$ \\
\hline IV 1d & $\begin{array}{l}231\left(15.7 \times 10^{4}\right), 293\left(4.35 \times 10^{4}\right), 312\left(4.13 \times 10^{4}\right) \\
320\left(4.2 \times 10^{4}\right), 330\left(4.3 \times 10^{4}\right), 345\left(4.14 \times 10^{4}\right)\end{array}$ & 368 & 453 & 85 & $4.9 \times 10^{-2}$ \\
\hline IV 1e & $\begin{array}{l}232\left(3.4 \times 10^{4}\right), 293\left(1.35 \times 10^{4}\right), 306\left(1.14 \times 10^{4}\right) \\
311\left(1.15 \times 10^{4}\right), 320\left(1.2 \times 10^{4}\right), 330\left(1.19 \times 10^{4}\right) \\
345\left(1.12 \times 10^{4}\right)\end{array}$ & 369 & 453 & 84 & $3.3 \times 10^{-3}$ \\
\hline IV $1 f$ & $\begin{array}{l}213\left(13.2 \times 10^{4}\right), 232\left(13.7 \times 10^{4}\right), 294\left(2.7 \times 10^{4}\right) \\
312\left(2.64 \times 10^{4}\right), 320\left(2.78 \times 10^{4}\right), 330\left(2.89 \times 10^{4}\right) \\
345\left(2.8 \times 10^{4}\right)\end{array}$ & 368 & 454 & 86 & $2.3 \times 10^{-1}$ \\
\hline IV $2 \mathbf{b}$ & $235\left(10.7 \times 10^{4}\right), 331\left(3.13 \times 10^{4}\right), 344\left(2.87 \times 10^{4}\right)$ & 369 & 454 & 85 & $2.6 \times 10^{-2}$ \\
\hline IV 2c & $241\left(1.83 \times 10^{4}\right), 317\left(1.54 \times 10^{4}\right), 373\left(4.16 \times 10^{3}\right)$ & 369 & 452 & 83 & $9.1 \times 10^{-2}$ \\
\hline IV $2 d$ & $\begin{array}{l}317\left(2.66 \times 10^{4}\right), 330\left(3 \times 10^{4}\right), 345\left(1.98 \times 10^{4}\right) \\
381\left(3 \times 10^{3}\right)\end{array}$ & 369 & 453 & 84 & $8 \times 10^{-2}$ \\
\hline IV $2 \mathbf{e}$ & $317\left(7.95 \times 10^{4}\right), 330\left(8.3 \times 10^{4}\right), 334\left(7 \times 10^{4}\right)$ & 368 & 453 & 85 & $2.4 \times 10^{-2}$ \\
\hline IV $2 f$ & $318\left(3.28 \times 10^{4}\right), 330\left(3.76 \times 10^{4}\right), 344\left(2.78 \times 10^{4}\right)$ & 369 & 453 & 84 & $6.2 \times 10^{-2}$ \\
\hline
\end{tabular}

Absorption maxima of the title compounds lie in the range of absorption maxima of naphthol and alkylated napthimide. It is therefore difficult to assign the light absorbing chromophore in the absence of delocalization aromatic $\pi$-electrons from one naphthyl ring at one end to the other naphthyl ring at the other end via 1,2, 3 -triazoles. To identify the light absorbing chromophore in the 1,4-disubstituted naphthoxymethyl-N-alkyl naphthalimide-1,2,3-triazoles, excitation and emission studies have been undertaken with spectrofluorophotometer.

Table 3. Electronic absorption and fluorescence data of $\alpha$-naphthol, 4-methoxy naphthol, naphthylpropargylicethers, 1,4disubstituted naphthyloxymethyl-N-alkylnaphthimido-1,2,3-triazoles in chloroform.

\begin{tabular}{|c|c|c|c|c|c|}
\hline Compound & $\lambda_{\mathrm{abs} \cdot \max }(\mathrm{nm})\left(\in, \mathrm{dm}^{2} \mathrm{~mol}^{1}{ }^{1}\right)$ & $\lambda_{\text {ex }}(\mathrm{nm})$ & $\lambda_{\text {flu.max }}(\mathrm{nm})$ & Stokes shift (nm) & $\Phi_{\mathrm{f}}$ \\
\hline I -1 & $323\left(2.8 \times 10^{3}\right)$ & 373 & 458 & 85 & $1.74 \times 10^{-3}$ \\
\hline I -2 & $333\left(1.12 \times 10^{4}\right), 374\left(1.75 \times 10^{3}\right)$ & 373 & 460 & 87 & $1.8 \times 10^{-3}$ \\
\hline III -1 & $320\left(4.11 \times 10^{2}\right), 352\left(4.33 \times 10^{1}\right)$ & 372 & 458 & 86 & $4.6 \times 10^{-6}$ \\
\hline III -2 & $244\left(4.7 \times 10^{3}\right), 316\left(1.29 \times 10^{3}\right), 361\left(2.96 \times 10^{2}\right)$ & 373 & 459 & 86 & $7.2 \times 10^{-5}$ \\
\hline IV $1 b$ & $\begin{array}{l}295\left(9 \times 10^{3}\right), 307\left(8.57 \times 10^{3}\right), 322\left(9.69 \times 10^{3}\right) \\
336\left(1.12 \times 10^{4}\right), 351\left(1.19 \times 10^{4}\right), 373\left(6.63 \times 10^{3}\right)\end{array}$ & 372 & 459 & 87 & $1.3 \times 10^{-2}$ \\
\hline IV 1c & $294\left(2.68 \times 10^{4}\right), 312\left(3.07 \times 10^{4}\right), 321\left(1.35 \times 10^{4}\right)$ & 395 & 434 & 39 & $2.4 \times 10^{-4}$ \\
\hline IV 1d & $\begin{array}{l}283\left(2 \times 10^{4}\right), 294\left(2.13 \times 10^{4}\right), 307\left(1.86 \times 10^{4}\right) \\
321\left(2.1 \times 10^{4}\right), 334\left(2.17 \times 10^{4}\right), 349\left(1.93 \times 10^{4}\right)\end{array}$ & 372 & 433 & 61 & $5.7 \times 10^{-3}$ \\
\hline IV 1e & $\begin{array}{l}294\left(1.64 \times 10^{4}\right), 307\left(1.37 \times 10^{4}\right), 321\left(1.47 \times 10^{4}\right) \\
335\left(1.49 \times 10^{4}\right), 350\left(1.35 \times 10^{4}\right)\end{array}$ & 372 & 457 & 85 & $2.8 \times 10^{-3}$ \\
\hline IV $1 f$ & $\begin{array}{l}295\left(2.52 \times 10^{4}\right), 307\left(2.29 \times 10^{4}\right), 321\left(2.65 \times 10^{4}\right) \\
334\left(2.84 \times 10^{4}\right), 349\left(2.55 \times 10^{4}\right)\end{array}$ & 370 & 459 & 89 & $9.5 \times 10^{-2}$ \\
\hline IV $2 \mathbf{b}$ & $241\left(6.29 \times 10^{4}\right), 283\left(1.6 \times 10^{4}\right), 336\left(3.85 \times 10^{4}\right)$ & 372 & 457 & 85 & $2.2 \times 10^{-1}$ \\
\hline IV 2c & $244\left(3.26 \times 10^{4}\right), 320\left(2.66 \times 10^{4}\right)$ & 372 & 458 & 86 & $4.5 \times 10^{-2}$ \\
\hline IV 2d & $241\left(10 \times 10^{4}\right), 332\left(4.3 \times 10^{4}\right)$ & 372 & 459 & 87 & $5.2 \times 10^{-2}$ \\
\hline IV 2 e & $284\left(2.1 \times 10^{4}\right), 332\left(3.59 \times 10^{4}\right)$ & 372 & 458 & 86 & $4.7 \times 10^{-2}$ \\
\hline IV $2 f$ & $242\left(6.7 \times 10^{4}\right), 333\left(3.83 \times 10^{4}\right)$ & 372 & 457 & 82 & $1.8 \times 10^{-2}$ \\
\hline
\end{tabular}


Table 4. Electronic absorption and fluorescence data of $\alpha$-naphthol, 4-methoxy naphthol, naphthylpropargylicethers, 1,4disubstituted naphthyloxy methyl-N-alkylnaphthimido-1,2,3-triazoles in methanol.

\begin{tabular}{|c|c|c|c|c|c|}
\hline Compound & $\lambda_{\text {abs } \cdot \max }(\mathrm{nm})\left(\in, \mathrm{dm}^{2} \mathrm{~mol}^{-1}\right)$ & $\lambda_{\text {ex }}(\mathrm{nm})$ & $\lambda_{\text {flu.max }}(\mathrm{nm})$ & Stokes shift $(\mathrm{nm})$ & $\Phi_{\mathrm{f}}$ \\
\hline I -1 & $232\left(2.4 \times 10^{4}\right), 295\left(4.3 \times 10^{3}\right), 323\left(2.4 \times 10^{3}\right)$ & 368 & 429 & 61 & $4.7 \times 10^{-4}$ \\
\hline I -2 & $244\left(4.3 \times 10^{3}\right), 321\left(1.1 \times 10^{3}\right), 332\left(1 \times 10^{3}\right)$ & 368 & 429 & 61 & $6 \times 10^{-5}$ \\
\hline III -1 & $227\left(5 \times 10^{3}\right), 289\left(1 \times 10^{3}\right), 319\left(3.23 \times 10^{2}\right)$ & 367 & 429 & 62 & $4.3 \times 10^{-7}$ \\
\hline III -2 & $242\left(6.2 \times 10^{3}\right), 315\left(1.6 \times 10^{3}\right), 328\left(1.4 \times 10^{3}\right)$ & 369 & 429 & 60 & $2.4 \times 10^{-4}$ \\
\hline IV $1 \mathrm{~b}$ & $\begin{array}{l}207\left(1.2 \times 10^{5}\right), 230\left(4.55 \times 10^{4}\right), 292\left(6.63 \times 10^{3}\right) \\
306\left(8.57 \times 10^{3}\right), 320\left(6.22 \times 10^{3}\right), 343\left(6.33 \times 10^{3}\right)\end{array}$ & 371 & 429 & 58 & $2.6 \times 10^{-3}$ \\
\hline IV 1c & $\begin{array}{l}222\left(7.76 \times 10^{4}\right), 292\left(2.75 \times 10^{4}\right), 312\left(3.13 \times 10^{4}\right), \\
320\left(3.3 \times 10^{4}\right)\end{array}$ & 368 & 428 & 60 & $5.3 \times 10^{-4}$ \\
\hline IV 1d & $\begin{array}{l}210\left(14.3 \times 10^{4}\right), 231\left(12.6 \times 10^{4}\right), 291\left(1.96 \times 10^{4}\right) \\
306\left(1.6 \times 10^{4}\right), 320\left(1.89 \times 10^{4}\right) \\
334\left(2 \times 10^{4}\right), 343\left(1.95 \times 10^{4}\right)\end{array}$ & 369 & 429 & 60 & $6.6 \times 10^{-2}$ \\
\hline IV 1e & $\begin{array}{l}227\left(3.69 \times 10^{4}\right), 292\left(1.6 \times 10^{4}\right), 306\left(1.29 \times 10^{4}\right) \\
312\left(1.23 \times 10^{4}\right), 320\left(1.42 \times 10^{4}\right) \\
333\left(1.44 \times 10^{4}\right), 343\left(1.38 \times 10^{4}\right)\end{array}$ & 350 & 429 & 79 & $1.8 \times 10^{-3}$ \\
\hline IV 1f & $\begin{array}{l}214\left(13.2 \times 10^{4}\right), 231\left(13.5 \times 10^{4}\right), 293\left(2 \times 10^{4}\right) \\
306\left(1.78 \times 10^{4}\right), 320\left(2.18 \times 10^{4}\right) \\
333\left(2.38 \times 10^{4}\right), 343\left(2.27 \times 10^{4}\right)\end{array}$ & 367 & 435 & 68 & $6.5 \times 10^{-2}$ \\
\hline IV $2 \mathbf{b}$ & $\begin{array}{l}209\left(9.3 \times 10^{4}\right), 233\left(11.8 \times 10^{4}\right), 333\left(3.44 \times 10^{4}\right) \\
343\left(3.27 \times 10^{4}\right)\end{array}$ & 347 & 401 & 54 & $2.6 \times 10^{-2}$ \\
\hline IV 2c & $209\left(12.3 \times 10^{4}\right), 242\left(2 \times 10^{4}\right), 318\left(2.12 \times 10^{4}\right)$ & 369 & 429 & 60 & $2.2 \times 10^{-2}$ \\
\hline IV 2d & $\begin{array}{l}210\left(14.4 \times 10^{4}\right), 234\left(9.93 \times 10^{4}\right), 330\left(2.97 \times 10^{4}\right), \\
343\left(2.2 \times 10^{4}\right)\end{array}$ & 369 & 428 & 59 & $4.3 \times 10^{-2}$ \\
\hline IV $2 e$ & $\begin{array}{l}209\left(15.2 \times 10^{4}\right), 234\left(10 \times 10^{4}\right), 331\left(3 \times 10^{4}\right) \\
343\left(2.1 \times 10^{4}\right)\end{array}$ & 368 & 429 & 61 & $2.3 \times 10^{-2}$ \\
\hline IV $2 f$ & $\begin{array}{l}212\left(9.69 \times 10^{4}\right), 234\left(10 \times 10^{4}\right), 331\left(3 \times 10^{4}\right), \\
343\left(1.2 \times 10^{4}\right)\end{array}$ & 369 & 429 & 60 & $2.2 \times 10^{-2}$ \\
\hline
\end{tabular}

Table 5. Electronic absorption and fluorescence data of $\alpha$-naphthol, 4-methoxy naphthol , naphthylpropargylicethers, 1,4disubstituted naphthyloxymethyl-N-alkyl naphthimido-1,2,3-triazoles in dimethylformamide.

\begin{tabular}{llcccc}
\hline Compound & $\lambda_{\text {abs } \cdot \max }(\mathrm{nm})\left(\in, \mathrm{dm}^{2} \mathrm{~mol}^{-1}\right)$ & $\lambda_{\text {ex }}(\mathrm{nm})$ & $\lambda_{\text {flu.max }}(\mathrm{nm})$ & Stokes shift $(\mathrm{nm})$ & $\Phi_{\mathrm{f}}$ \\
\hline I -1 & $298\left(5 \times 10^{3}\right), 310\left(4.27 \times 10^{3}\right), 324\left(3.43 \times 10^{3}\right)$ & 373 & 434 & 61 & $7.3 \times 10^{-6}$ \\
I -2 & $278\left(7.3 \times 10^{3}\right), 322\left(1.4 \times 10^{4}\right), 355\left(1.36 \times 10^{4}\right)$ & 373 & 434 & 61 & $2 \times 10^{-3}$ \\
III -1 & $260\left(6.68 \times 10^{2}\right), 292\left(1.1 \times 10^{3}\right), 321\left(3.9 \times 10^{2}\right)$ & 374 & 434 & 60 & $6.2 \times 10^{-7}$ \\
III -2 & $227\left(1.18 \times 10^{3}\right), 317\left(1.79 \times 10^{3}\right), 330\left(1.69 \times 10^{3}\right)$ & 374 & 434 & 60 & $2.7 \times 10^{-4}$ \\
IV 1b & $295\left(6.53 \times 10^{3}\right), 307\left(6.5 \times 10^{3}\right) 321\left(6.94 \times 10^{3}\right)$, & 372 & 433 & 61 & $3.4 \times 10^{-2}$ \\
& $334\left(6.73 \times 10^{3}\right)$ & & & & \\
IV 1c & $295\left(2.49 \times 10^{4}\right), 312\left(2.9 \times 10^{4}\right), 320\left(2.92 \times 10^{4}\right)$ & 373 & 434 & 61 & $1.5 \times 10^{-3}$ \\
IV 1d & $295\left(1.87 \times 10^{4}\right), 295\left(1.7 \times 10^{4}\right)$, & 372 & 433 & 61 & $3.6 \times 10^{-2}$ \\
& $321\left(1.95 \times 10^{4}\right), 334\left(1.98 \times 10^{4}\right)$ & & & & \\
IV 1e & $295\left(1.63 \times 10^{4}\right), 295\left(1.42 \times 10^{4}\right)$, & 373 & 433 & 60 & $3.5 \times 10^{-2}$ \\
& $313\left(1.4 \times 10^{4}\right), 321\left(1.49 \times 10^{4}\right), 334\left(1.42 \times 10^{4}\right)$ & & & & \\
IV 1f & $295\left(2 \times 10^{4}\right), 307\left(2 \times 10^{4}\right), 322\left(2.28 \times 10^{4}\right)$, & 372 & 434 & 62 & $6.98 \times 10^{-2}$ \\
& $334\left(2.39 \times 10^{4}\right), 343\left(2.2 \times 10^{4}\right)$ & 372 & 434 & 62 & $7.5 \times 10^{-2}$ \\
IV 2b & $333\left(3.31 \times 10^{4}\right), 342\left(2 \times 10^{4}\right)$ & 373 & 433 & 60 & $4.6 \times 10^{-2}$ \\
IV 2c & $320\left(2.09 \times 10^{4}\right), 342\left(5.9 \times 10^{3}\right)$ & 373 & 434 & 61 & $4.8 \times 10^{-2}$ \\
IV 2d & $322\left(3.14 \times 10^{4}\right), 344\left(2 \times 10^{4}\right)$ & 373 & 433 & 60 & $3.43 \times 10^{-2}$ \\
IV 2e & $332\left(3.2 \times 10^{4}\right), 344\left(2.1 \times 10^{4}\right)$ & 373 & 434 & 61 & $9.4 \times 10^{-2}$ \\
IV 2f & $332\left(3.24 \times 10^{4}\right), 344\left(8.8 \times 10^{3}\right)$ & & &
\end{tabular}


Table 6. Electronic absorption and fluorescence data of $\alpha$-naphthol, 4-methoxy naphthol, naphthylpropargylicethers, 1,4disubstituted naphthyloxymethyl-N-alkyl naphthimido-1,2,3-triazoles in water.

\begin{tabular}{llcccc}
\hline Compound & $\lambda_{\text {abs } \cdot \max }(\mathrm{nm})\left(\in, \mathrm{dm}^{2} \mathrm{~mol}^{-1}\right)$ & $\lambda_{\text {ex }}(\mathrm{nm})$ & $\lambda_{\text {flu.max }}(\mathrm{nm})$ & Stokes shift $(\mathrm{nm})$ & $\Phi_{\mathrm{f}}$ \\
\hline I -1 & $291\left(4 \times 10^{3}\right), 321\left(2 \times 10^{3}\right), 361\left(2.3 \times 10^{2}\right)$ & 362 & 451 & 89 & $1.2 \times 10^{-4}$ \\
I -2 & $241\left(3.78 \times 10^{4}\right)$, & 374 & 431 & 57 & $1.45 \times 10^{-4}$ \\
& $318\left(9.3 \times 10^{3}\right), 329\left(8.77 \times 10^{3}\right)$ & & & & \\
III -1 & $287\left(9.17 \times 10^{2}\right), 319\left(2.95 \times 10^{2}\right)$, & 362 & 462 & 100 & $6.3 \times 10^{-6}$ \\
& $361\left(1.17 \times 10^{1}\right)$ & & & & \\
III -2 & $240\left(4.92 \times 10^{3}\right), 304\left(1.29 \times 10^{3}\right)$, & 360 & 413 & 53 & $1.39 \times 10^{-4}$ \\
& $313\left(1.3 \times 10^{3}\right)$ & & & & \\
IV 1b & $350\left(9.8 \times 10^{4}\right)$ & 346 & 395 & 49 & $2 \times 10^{-3}$ \\
IV 1c & $320\left(1.95 \times 10^{3}\right)$ & 321 & 384 & 63 & $1.5 \times 10^{-4}$ \\
IV 1d & $358\left(1.43 \times 10^{4}\right)$ & 343 & 384 & 41 & $2.5 \times 10^{-4}$ \\
IV 1e & $320\left(7.3 \times 10^{2}\right), 344\left(7 \times 10^{2}\right)$ & 344 & 390 & 46 & $5.93 \times 10^{-3}$ \\
IV 1f & $348\left(5.3 \times 10^{1}\right)$ & 343 & 395 & 52 & $1 \times 10^{-5}$ \\
IV 2b & $293\left(7.8 \times 10^{3}\right), 344\left(3.2 \times 10^{4}\right)$ & 344 & 384 & 40 & $1.92 \times 10^{-4}$ \\
IV 2c & $321\left(2.5 \times 10^{3}\right)$ & 322 & 384 & 62 & $1.32 \times 10^{-4}$ \\
IV 2d & $332\left(2.13 \times 10^{2}\right), 343\left(1.42 \times 10^{2}\right)$ & 341 & 386 & 45 & $8.6 \times 10^{-5}$ \\
IV 2e & $354\left(6.23 \times 10^{3}\right)$ & 355 & 406 & 51 & $1.99 \times 10^{-3}$ \\
IV 2f & $234\left(3.68 \times 10^{3}\right), 343\left(1.23 \times 10^{3}\right)$ & 345 & 392 & 47 & $2.96 \times 10^{-4}$ \\
\hline
\end{tabular}

\subsection{Fluorescence study}

Fluorescence spectra of IV 1d, IV 1e, IV 1f, IV 2d and IV $2 \mathrm{f}$ in cyclohexane are given in figure 3. Similarly, fluorescence spectra of IV 1f in cyclohexane, chloroform, methanol, DMF and water are shown in figure 4. From the figure 3 it can be observed that emission spectra of IV If appear similar to a mirror image of its absorption spectrum.

Similar observation was made with the other compounds in all the investigated solvents. Emission data obtained in the present study is tabulated in tables 2 to 6 . The light absorbing chromophore was not established

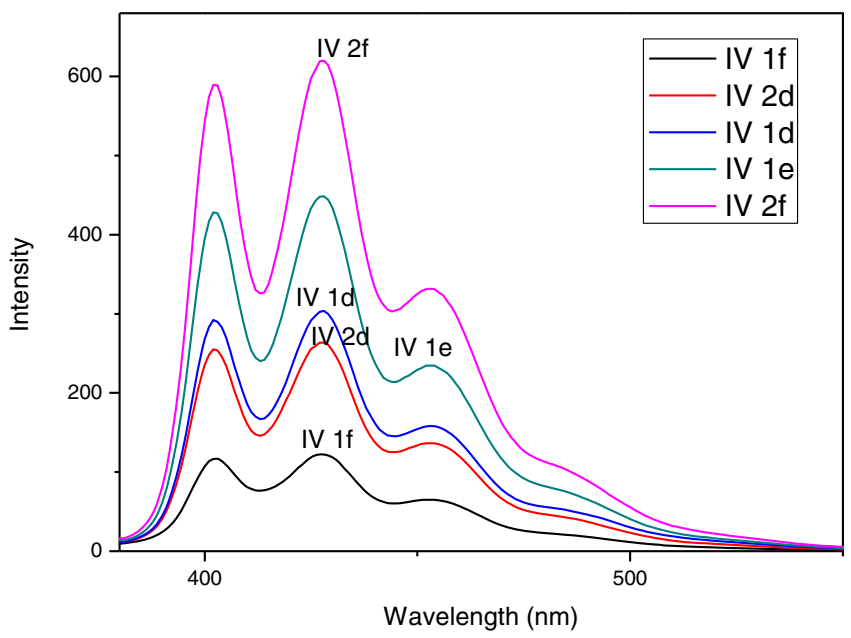

Figure 3. Fluorescence spectra of IV $2 \mathrm{f} 2.6 \times 10^{-5} \mathrm{M}$, IV 1e $1 \times 10^{-4} \mathrm{M}$, IV $1 \mathrm{~d} 9.8 \times 10^{-6} \mathrm{M}$, IV $2 \mathrm{~d} 1.41 \times 10^{-5} \mathrm{M}$ and IV $1 \mathrm{f} 1.88 \times 10^{-5} \mathrm{M}$ in cyclohexane. by absorption spectroscopy as both the naphthalene rings at 1,4-position of the triazole absorb in the same region. However, from the excitation and emission maxima, napthol is the assigned light absorbing and emitting chromophore but not naphthalimide. The excitation and emission wavelength maxima in the disubstituted triazole compounds are close to that of naphthol rather than naphthalimide. The excitation and emission wavelength maxima of naphthimides are $<340$ and $400 \mathrm{~nm}$, respectively. However, in the present 1,4-disubstituted naphthoxymethyl-N-alkyl naphthalimide-1,2,3-triazoles, the excitation and emission maxima are in the region 365 to $370 \mathrm{~nm}$ and 400 to $460 \mathrm{~nm}$, respectively. Therefore, the light absorbing

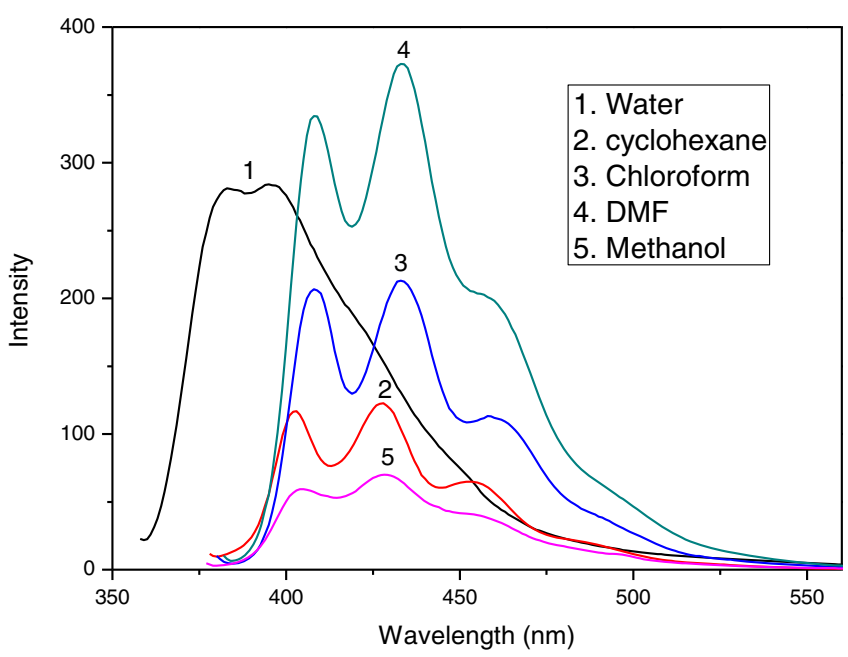

Figure 4. Fluorescence spectra of IV 1f $1.88 \times 10^{-5} \mathrm{M}$ in different solvents. 
chromophore is the naphthol moiety rather than the naphthimide part of 1,2,3-triazole. The absorption maxima and the emission maxima of the methoxysubstituted naphthoxytriazoles (IV 2) are similar to that of the simple naphthoxy-substituted triazole (IV 1) derivatives. This is possibly due to the absence of delocalization of $\pi$-electrons of the positive mesomerism of methoxy group to an extent of influencing the absorption of light. Both the substituted naphthalene rings at 1,4-position of 1,2,3-triazoles are neither in conjugation with one another, nor with the triazole ring as the naphthoxy part is insulated by $-\mathrm{OCH}_{3}$ and the naphthalimide group by alkyl chain. Therefore, it can be concluded that in the absence of conjugation between the three absorbing chromophores, i.e., 1-naphthyloxy, 1,2,3-triazole and naphthalimide, the light absorbing chromophore is the naphthyloxychromophore.

The naphthoxymethylalkylimido-1,2,3-triazoles are moderately fluorescent compounds, exhibiting little solvent and substituent effects on the quantum yield and emission maxima. A closer look at tables 2 to 6 reveals that the order of quantum yield is IV $>$ I $2>I 1>I I I$ $2>$ III 1. A triazole ring added on to naphtholproporgyl ether improved the light emission; the methoxy substituent in naphthol also enhances the radiative emission. However, proporgylation on the oxygen of 1-naphthol quenched the fluorescence. The length of the alkyl chain between the triazole and naphthimide did not bring any considerable changes in spectral properties of the 1,4-disubstituted naphthoxymethyl-Nalkyl naphthalimide-1,2,3-triazoles. Aprotic polar solvent, DMF enhanced the quantum yield while the protic polar solvents quenched the fluorescence. The effect of the solvent on the quantum yield of a given compound follows the order: DMF $>$ cyclohexane $>$ chloroform $>$ methanol $>$ water. Aqueous medium is the more quenching medium. Similarly, the effect of solvent on fluorescence emission maximum and excitation maxima is not the same for all the compounds. However, a broad generalization can be made based on the spectral data presented in the tables 2 to 6 . The effect of solvent on the emission maxima varies in the order: chloroform $>$ cyclohexane $>$ methanol $>$ DMF $>$ water. The water is unique in the sense that it is the most quenching and hypsochromic medium than the remaining media. This may be due to the fact that absorption and emission maxima in water are attributed to the naphthalimide part rather than naphthol chromophore, unlike in the remaining solvents. ${ }^{28}$ The Stokes shift exhibits linearity neither with the solvent polarity nor to the length of the alkyl chain, indicating that the absorbing and emitting chromophore is the naphthoyloxy moiety.

\section{Conclusions}

Synthesis of a few 1,4-disubstituted naphthoxymethyl$\mathrm{N}$-alkylnaphthalimide-1,2,3-triazols is reported. The structure of these triazoles was established by spectral studies. It is proved that electronic excitation and emission techniques are superior techniques over the electronic absorption spectral technique to identify the light absorbing chromophore. $\mathrm{Sp}^{3}$-hybridized linker atoms such as nitrogen, oxygen and carbon in between the triazole ring and the naphthalene moieties at 1,4-position prevent extensive delocalization of $\pi$-electrons leading to little change in the absorption and emission properties of 1,4-disubstituted naphthoxymethyl-Nalkylnaphthalimide-1,2,3-triazols when compared with 1-naphthol.

\section{Supplementary information}

${ }^{1} \mathrm{H},{ }^{13} \mathrm{C}$ spectra, mass spectra, spectral data, fluorescence spectra and tables are submitted as supplementary information.

\section{Acknowledgements}

One of the authors $\mathrm{J}$ Ramchander thanks Osmania University, Hyderabad for providing financial support under faculty development programme.

\section{References}

1. Kolb H C, Finn M G and Sharpless K B 2001 Angew. Chem. Int. Ed. 402004

2. Tornoe C W, Christensen C and Meldal M 2002 J. Org. Chem. 673057

3. David O, Maisonneuve S and Xie J 2007 Tetrahedron. Lett. 486527

4. Huang S, Clark R J and Zhu L 2007 Org. Lett. 94999

5. Maisonneuve S, Fang Q and Xie J 2008 Tetrahedron 64 8716

6. Garcia L, Maisonneuve S, Xie J, Guillot R, Dorlet P, Riviere E, Desmadril M, Lambert F and Policar C 2010 Inorg. Chem. 497282

7. Maity D and Govindaraju T 2010 Chem. Commun. 46 4499

8. Garcia L, Maisonneuve S, Marcu J O S, Guillot R, Lambert F, Xie J and Policar C 2011 Inorg. Chem. 50 11353

9. Wu Y, Dong Y, Li J, Huang X, Cheng Y and Zhu C 2011 Chem. An. Asian J. 62725

10. Lau Y H, Price J R, Todd M H and Rutledge P J 2011 Chem. Eur. J. 172850

11. Ast S, Rutledge P J and Todd M H 2012 Eur. J. Inorg. Chem. 345611 
12. Lau Y H, Rutledge $\mathrm{P}$ J, Watkinson $\mathrm{M}$ and Todd $\mathrm{M} \mathbf{H}$ 2011 Chem. Soc. Rev. 402848

13. Brombosz S M, Appleton A L, Zappas A J 2nd and Bunz U H F 2010 Chem. Commun. 461419

14. Lee J, Shizu K, Tanaka H, Nomura H, Yasuda T and Adachi C 2013 J. Mater. Chem. C 14599

15. Ruan Y-B, Yu Y, Li C, Bogliotti N, Tang J and Xie J 2013 Tetrahedron 694603

16. Jia T-J, Cao W, Zheng X-J and Jin L-P 2013 Tetrahedron Lett. 543471

17. Czirok J B, Bojtar M, Hessz D, Baranyai P, Drahos L, Kubinyi M and Bitter I 2013 Sensors and Actuators B 182280

18. Bag S S, Kundu R and Talukdar S 2012 Tetrahedron Lett. 535875

19. Prasad D J, Ashok M, Karegoudar P, Poojary B, Holla B S and Kumari N S 2009 Eur. J. Med. Chem. 44 551
20. Tornoe C W, Christensen C and Meldal M 2002 J. Org. Chem. 673057

21. Holla B S, Veerendra B, Shivananda M K and Poojary B 2003 Eur. J. Med. Chem. 38759

22. Amir M and Shikha K 2004 Eur. J. Med. Chem. 39 535

23. Almasirad A, Tabatabai S A, Faizi M, Kebriaeezadeh A, Mehrabi N, Dalvandi A and Shafiee A 2004 Bioorg. Med. Chem. Lett. 146057

24. Kolb H C, Finn M G and Sharpless K B 2001 Angew. Chem. Int. Ed. 402004

25. Piplani P, Malik R, Kaur B and Kaplish 2012 Med. Chem. Res. 211771

26. Kolb H C and Sharpless K B 2003 Drug Discov. Today 81128

27. Ramchander J 2012 Der Pharma. Chemica 4694

28. Sheshashena Reddy T and Ram Reddy A 2012 J. Photochem. Photobiol. A 22751 